\title{
Computationally Efficient Field-Theoretic Simulations for Block Copolymer Melts
}

\author{
T. M. Beardsley, ${ }^{\dagger}$ R. K. W. Spencer, ${ }^{\dagger}$ and M. W. Matsen ${ }^{*,+}+, \uparrow$ \\ $\dagger$ †epartment of Physics $\&$ Astronomy, University of Waterloo, Waterloo, Ontario, Canada \\ $\ddagger$ Department of Chemical Engineering, University of Waterloo, Waterloo, Ontario, Canada \\ ฯ Waterloo Institute for Nanotechnology, University of Waterloo, Waterloo, Ontario, \\ Canada \\ E-mail: mwmatsen@uwaterloo.ca
}

\begin{abstract}
Field-theoretic simulations (FTS) provide fluctuation corrections to self-consistent field theory (SCFT) by simulating its field-theoretic Hamiltonian rather than applying the saddle-point approximation. Although FTS work well for ultrahigh molecular weights, they have struggled with experimentally relevant values. Here, we consider FTS for two-component (i.e., AB-type) melts, where the composition field fluctuates but the saddle-point approximation is still applied to the pressure field that enforces incompressibility. This results in real-valued fields, thereby allowing for conventional simulation methods. We discover that Langevin simulations are one to two orders of magnitude faster than previous Monte Carlo simulations, which permits us to accurately calculate the order-disorder transition of symmetric diblock copolymer melts at realistic molecular weights. This remarkable speedup will, likewise, facilitate FTS for more complicated block copolymer systems, which might otherwise be unfeasible with traditional particle-based simulations.
\end{abstract}




\section{Introduction}

Self-consistent field theory $(\mathrm{SCFT})^{1}$ has proven to be a remarkably successful theory for predicting the equilibrium behavior of block polymer melts. ${ }^{2,3}$ It is generally applied to the standard Gaussian-chain model $(\mathrm{GCM}),{ }^{4}$ which treats a melt as an incompressible system of elastic threads interacting by contact forces. The universality of block copolymer phase

behavior implies that all systems reduce to the GCM at high molecular weights, ${ }^{5,6}$ where polymers are many persistence lengths long and much larger than the range of their interactions. The prototypical system is the diblock copolymer melt consisting of $n$ linear polymers, each with $N_{A}$ A-type segments joined to $N_{B}$ B-type segments, where the strength of the A-B interactions is controlled by an effective Flory-Huggins $\chi$ parameter. (Note that we apply the usual convention of defining segments based on a common volume of $\rho_{0}^{-1}$.)

In SCFT, the particle-based GCM is transformed to a mathematically equivalent fieldbased model, involving a composition field, $W_{-}(\mathbf{r})$, that couples to the difference between $\mathrm{A}$ and $\mathrm{B}$ concentrations, $\phi_{-}(\mathbf{r})$, and a pressure field, $W_{+}(\mathbf{r})$, that couples to the total concentration, $\phi_{+}(\mathbf{r})$, in a system of non-interacting polymers. The field-based Hamiltonian takes the form ${ }^{2,3}$

$$
\begin{aligned}
\frac{H_{\mathrm{p}}\left[W_{-}, W_{+}\right]}{k_{B} T}= & -n \ln Q\left[W_{-}, W_{+}\right]+ \\
& \rho_{0} \int\left(\frac{W_{-}^{2}(\mathbf{r})}{\chi_{b}}-W_{+}(\mathbf{r})\right) d \mathbf{r}
\end{aligned}
$$

where $\chi_{b}$ is a bare interaction parameter and $Q\left[W_{-}, W_{+}\right]$is the partition function for a single molecule in the system of non-interacting polymers. The total partition function of the melt is evaluated using the saddle-point approximation. The saddle point, denoted by $w_{-}(\mathbf{r})$ and $w_{+}(\mathbf{r})$, is obtained by solving the self-consistent conditions $g_{-}\left[w_{-}, w_{+}\right]=g_{+}\left[w_{-}, w_{+}\right]=0$, 
where

$$
\begin{aligned}
& g_{-}\left[W_{-}, W_{+}\right]=\phi_{-}\left[W_{-}, W_{+}\right]+\frac{2}{\chi_{b}} W_{-} \\
& g_{+}\left[W_{-}, W_{+}\right]=\phi_{+}\left[W_{-}, W_{+}\right]-1
\end{aligned}
$$

Once the saddle point is located, the free energy is given by $H_{p}\left[w_{-}, w_{+}\right]$. The self-consistent field conditions generally have multiple solutions corresponding to different metastable phases; the one of lowest free energy represents the stable phase. In SCFT, $\chi$ and $\chi_{b}$ are the same.

Although SCFT is found to be accurate for ordered morphologies, ${ }^{7}$ its treatment of the disordered phase as a homogeneous mixture of $\mathrm{A}$ and $\mathrm{B}$ segments is wholly inaccurate. ${ }^{8}$ As a consequence, there are large fluctuation corrections to the order-disorder transition (ODT). The size of the corrections is controlled by the invariant polymerization index, $\bar{N} \equiv$ $a^{6} \rho_{0}^{2} N$, where $a$ is the statistical length of the segments. (Note that we limit our attention to conformationally symmetric systems, where both components have the same a.) For symmetric diblock copolymers (i.e., $N_{A}=N_{B}$ ), the ODT is given by

$$
(\chi N)_{\mathrm{ODT}}=10.495+41.0 \bar{N}^{-1 / 3}+123.0 \bar{N}^{-0.56}
$$

where the first term is the SCFT prediction, the second is the well-known Fredrickson-Helfand (F-H) correction, ${ }^{9}$ and the third is an additional correction obtained from particle-based simulations. ${ }^{10}$

In field-theoretic simulations (FTS), one simulates $H_{p}\left[W_{-}, W_{+}\right]$rather than applying the saddle-point approximation of SCFT. Ideally, both fields would be allowed to fluctuate, but this is complicated by the fact that $W_{+}(\mathbf{r})$ takes on imaginary values. Fredrickson and coworkers have dealt with this by performing complex Langevin simulations (CL-FTS). ${ }^{11-14}$ Another strategy is to assume that the composition fluctuations are dominant, and therefore continue to apply the saddle-point approximation for $W_{+}(\mathbf{r})$. This is done by simulating $H_{p}\left[W_{-}, w_{+}\right]$, where $w_{+}(\mathbf{r})$ satisfies $g_{+}\left[W_{-}, w_{+}\right]=0$. As it turns out, $w_{+}(\mathbf{r})$ is real valued, 
which then allows for the use of conventional simulation methods. Most studies apply Monte Carlo dynamics (MC-FTS), ${ }^{15-19}$ but a couple have implemented standard Langevin dynamics $(\mathrm{L}-\mathrm{FTS}) \cdot{ }^{20,21}$

The simulations are typically performed in a cubic simulation box of volume $V=L^{3}$ with periodic boundaries. The fields are represented on a discrete grid with $m$ points in each direction, separated by a uniform spacing of $\Delta$ (i.e., $L=m \Delta$ ). One might expect the simulations to become increasingly accurate as $\Delta \rightarrow 0$, but instead this leads to an ultraviolet (UV) divergence that tends to disorder the melt. Olvera de la Cruz et al. ${ }^{22}$ showed that this could be compensated for by renormalizing the $\chi$ parameter. Although this works well at large $\bar{N},{ }^{17}$ it begins to fail as the experimentally relevant regime is approached. ${ }^{18}$ We recently showed ${ }^{19}$ that the UV divergence can be dealt with by defining an effective $\chi$ parameter using the Morse calibration ${ }^{5-7,10,23}$ devised for particle-based simulations. For the case of FTS,

$$
\chi=\frac{z_{\infty} \chi_{b}+c_{1} \chi_{b}^{2}}{1+c_{2} \chi_{b}}
$$

where

$$
z_{\infty}=1+\frac{2.332654}{a^{2} \rho_{0} \Delta}
$$

is the fraction of intermolecular contacts in an athermal melt. ${ }^{19}$ The remaining coefficients, $c_{1}$ and $c_{2}$, are determined by fitting the structure function, $S(k)$, from FTS to renormalized-one loop (ROL) predictions. ${ }^{24,25}$

Although this strategy resolved the UV divergence, the Monte Carlo dynamics used in ref 19 became relatively slow as $\bar{N}$ was reduced to realistic values. This was further exacerbated by the fact that the amplitude of the MC moves had to be reduced for increasing system size. It was suggested that this shortcoming might be overcome by switching to Langevin dynamics. Here, we investigate this possibility, in an effort to resolve the last major obstacle impeding FTS for realistic molecular weights. ${ }^{26}$ 


\section{Statistical mechanics of non-interacting polymers}

The most computational part of field-theoretic calculations is the statistical mechanics for the system of non-interacting polymers in the $W_{-}(\mathbf{r})$ and $W_{+}(\mathbf{r})$ fields. ${ }^{1-4}$ It requires the calculation of a partial partition function, $q(\mathbf{r}, t)$, for the first $t$ segments of a polymer with the $t^{\prime}$ th segment constrained at position $\mathbf{r}$. This function satisfies the diffusion equation

$$
\frac{\partial q}{\partial t}=\frac{a^{2}}{6} \nabla^{2} q-\left(W_{+}+\gamma W_{-}\right) q
$$

subject to the initial condition $q(\mathbf{r}, 0)=1$. Here, we define $\gamma(t)$ to be +1 for the A block and -1 for the B block. One also requires an analogous function, $q^{\dagger}(\mathbf{r}, t)$, for the last $N-t$ segments. It is obtained by integrating eq 7 with one side multiplied by -1 , backwards in $t$, starting from $q^{\dagger}(\mathbf{r}, N)=1$. Once the two partial partition functions are known, the single-chain partition function is obtained from

$$
Q=\int q(\mathbf{r}, t) q^{\dagger}(\mathbf{r}, t) d \mathbf{r}
$$

The above integral can be evaluated using any value of $t$, and so one typically chooses $t=N$. The composition and total concentration of the noninteracting polymers are

$$
\begin{aligned}
\phi_{-}(\mathbf{r}) & =\frac{n}{\rho_{0} Q} \int_{0}^{N} \gamma(t) q(\mathbf{r}, t) q^{\dagger}(\mathbf{r}, t) d t \\
\phi_{+}(\mathbf{r}) & =\frac{n}{\rho_{0} Q} \int_{0}^{N} q(\mathbf{r}, t) q^{\dagger}(\mathbf{r}, t) d t
\end{aligned}
$$

respectively, where the number of polymers is given by $n=\rho_{0} V / N$.

We solve the diffusion eq 7 numerically using a pseudo-spectral method ${ }^{27}$ with Richardson extrapolation, ${ }^{28}$ and we evaluate the integrals in eqs 9 and 10 using the Simpson quadrature. The number of integration steps along the polymer contour serves as our definition of the polymerization, $N$. The grid resolution is chosen as $\Delta=a$, which is sufficiently large relative 
to the integration step for the polymers to behave as continuous chains. ${ }^{19}$ The segment density is set to $\rho_{0}=8 / a^{3}$, which results in an invariant polymerization index of $\bar{N}=64 N$.

\section{Langevin simulation algorithm}

In order to reduce the simulations to one fluctuating field, $W_{+}(\mathbf{r})$ is set to the saddle point, $w_{+}(\mathbf{r})$, which we obtain iteratively using Anderson mixing. ${ }^{29,30}$ The algorithm follows that of the SCFT calculations in ref $31 .^{32}$ Here, we apply the iterations until the self-consistent condition, $g_{+}\left[W_{-}, w_{+}\right]=0$, is satisfied to an error tolerance of $\varepsilon=10^{-4}$.

The remaining field, $W_{-}(\mathbf{r} ; \tau)$, is then evolved using Langevin dynamics. The previous L-FTS by Reister et al. ${ }^{20}$ studied the kinetics of macrophase separation in $\mathrm{A}+\mathrm{B}$ homopolymer blends, whereas we just use Langevin dynamics to produce a sequence of equilibrated configurations. Thus, there is no need for us to calibrate the time-like variable, $\tau$. The sequence (i.e., $\tau=k \delta \tau$ for $k=1,2,3, \ldots$ ) is generated using

$$
W_{-}(\mathbf{r} ; \tau+\delta \tau)=W_{-}(\mathbf{r} ; \tau)-\Lambda(\mathbf{r}, \tau) \delta \tau+\mathcal{N}(0, \sigma)
$$

where $\Lambda(\mathbf{r}, \tau)$ is a forcing term and $\mathcal{N}(0, \sigma)$ is random noise with a normal distribution of zero mean and variance

$$
\sigma^{2}=\frac{2 \delta \tau}{\Delta^{3} \rho_{0}}
$$

To improve accuracy, we apply the predictor-corrector algorithm. ${ }^{33}$ A predicted field, $W_{-}^{*}(\mathbf{r} ; \tau+$ $\delta \tau)$, is first evaluated using eq 11 with

$$
\Lambda^{*}=\left.g_{-}\left[W_{-}, w_{+}\right]\right|_{W_{-}(\mathbf{r} ; \tau)}
$$

and then a corrected field, $W_{-}(\mathbf{r} ; \tau+\delta \tau)$, is obtained with

$$
\Lambda=\frac{1}{2} \Lambda^{*}+\left.\frac{1}{2} g_{-}\left[W_{-}, w_{+}\right]\right|_{W_{-}^{*}(\mathbf{r} ; \tau+\delta \tau)}
$$


(Note that the predictor and corrector steps use the same random number.) As usual, the Langevin dynamics are applied for a sufficient amount of time (e.g., $10^{5} \delta \tau$ ) to allow the system to equilibrate, before observables are sampled, typically once every $10 \delta \tau$.
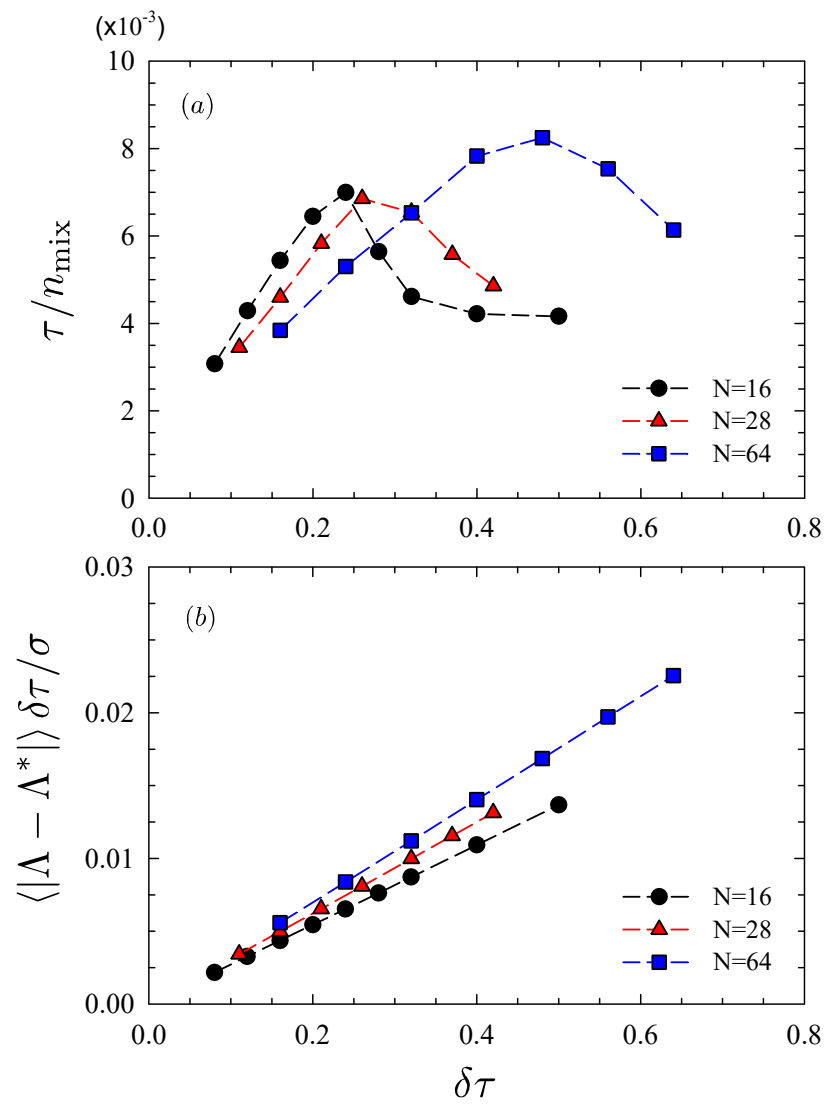

Figure 1: (a) Change in $\tau$ per Anderson mixing iteration and (b) measure of the relative difference in the corrector and predictor parts of the Langevin dynamics as a function of step size, $\delta \tau$, for different polymerizations, $N$. The peaks in plot a identify the optimum step sizes and the small differences between $\Lambda$ and $\Lambda^{*}$ in plot b ensure the accuracy of the predictor-corrector algorithm.

Normally, the time step, $\delta \tau$, is chosen as large as possible, limited only by the requirement of numerical accuracy. However, in our case, there is the additional consideration that the number of Anderson mixing iterations, $n_{\text {mix }}$, increases for larger steps. This leads to an optimum time step, as illustrated in Figure 1a for simulations near the ODT. The optimum value depends on $N$, but has no noticeable dependence on $m$. This latter fact leads to far superior scaling with respect to system size than was the case for previous MC-FTS. ${ }^{19}$

Of course, we still need to ensure that the finite size of $\delta \tau$ does not significantly impact 
the accuracy of the predictor-corrector algorithm. As a rule of thumb, the difference between $\Lambda^{*} \delta \tau$ and $\Lambda \delta \tau$ defined by eqs 13 and 14, respectively, should be small relative to the typical change in $W_{-}(\mathbf{r} ; \tau)$, the magnitude of which is given by the standard deviation of the random noise, $\sigma$. Figure $1 b$ illustrates that this condition is indeed well satisfied. In fact, we could probably drop the corrector step without any noticeable sacrifice in accuracy. Nevertheless, the computational cost of including the corrector step is minimal on account of the relatively few Anderson mixing iterations required, and so we choose to keep it.

\section{Morse Calibration}

Armed with the ability to simulate larger systems, we repeat the Morse calibration ${ }^{5,6,23}$ for the effective $\chi$ in eq 5 performed previously in ref 19. The fraction of intermolecular contacts,

$$
z_{\infty}=0.7084
$$

still obeys the analytical expression in eq 6 , and thus is unaffected. However, $c_{1}$ and $c_{2}$ are obtained from simulations of the structure function, ${ }^{20}$

$$
\frac{S(\mathbf{k})}{\rho_{0} N}=\frac{n}{\left(V \chi_{b}\right)^{2}}\left\langle\left|W_{-}(\mathbf{k})\right|^{2}\right\rangle-\frac{1}{2 \chi_{b} N}
$$

which is affected by the finite size, $m$, of the simulation box. In particular, its finite size restricts the wavevector to the discrete values: $\mathbf{k}=2 \pi(h, k, l) / m \Delta$, where $h, k$, and $l$ are integers. The consequence of this is illustrated in Figure 2, where the spherically-averaged $S(k)$ for $N=16$ from MC-FTS in a simulation box of $m=16$ is compared to that from L-FTS in a box of $m=32$. Although the previous MC-FTS results are accurate for most $\chi_{b} N$ values, a significant inaccuracy occurs at the highest segregations, where the number of wavevectors in the peak is relatively few.

Fitting the updated values of $S\left(k^{*}\right)$, expressed as a function of $\chi_{b}$, to the ROL predictions, 


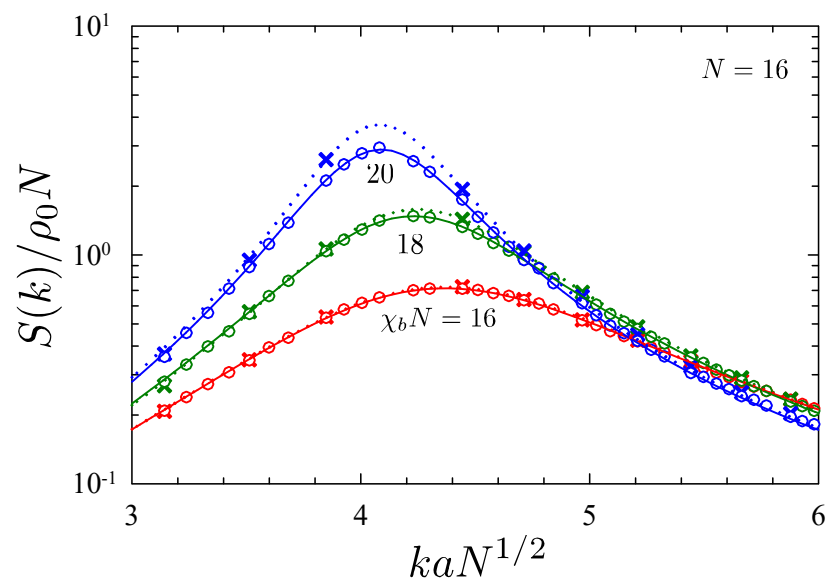

Figure 2: Structure function, $S(k)$, for molecules of polymerization $N=16$ calculated at different segregations, $\chi_{b} N$. Crosses denote previous MC-FTS results with a simulation box of $m=16,{ }^{19}$ while circles correspond to L-FTS with $m=32$. The curves are fits used to extract the peak, $S\left(k^{*}\right)$.

expressed as a function of $\chi$, gives

$$
c_{1}=1.246 \quad \text { and } \quad c_{2}=1.367
$$

The quality of the fit is illustrated in Figure 3, where the L-FTS results are plotted with symbols and the ROL predictions are given by solid curves. As a result of the partial saddlepoint approximation, the L-FTS results converge to the mean-field prediction from RPA (dashed line) ${ }^{34}$ at small $\chi N$. Consequently, the L-FTS are unable to reproduce the slight deviation predicted by ROL, which is well reproduced by particle-based simulations. ${ }^{5,6,10}$ Therefore, we limit our fit to the data points for $\chi N>7$. The inset of Figure 3 shows the resulting relationship between $\chi$ and $\chi_{b}$, as well as the linear approximation, $\chi \approx z_{\infty} \chi_{b}$, corresponding to the renormalization first used in ref 17 . The difference in $\chi$ from the previous calibration in ref 19 is slight, but nevertheless large enough to have a noticable effect. 


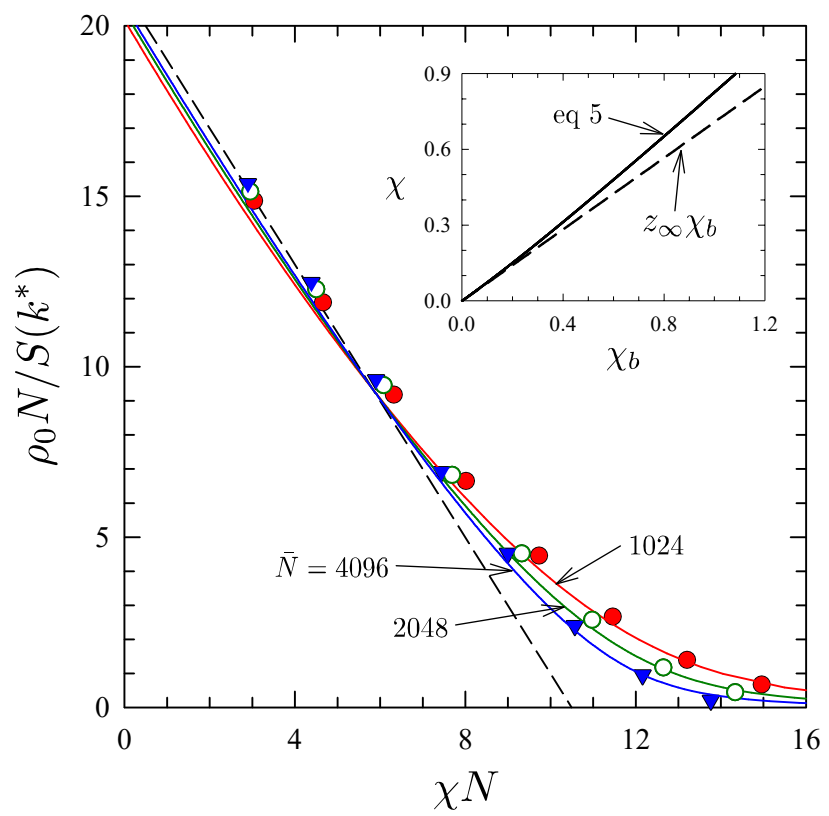

Figure 3: Inverse peak height of the structure function, $S^{-1}\left(k^{*}\right)$, plotted in terms of the effective $\chi$ parameter for different invariant polymerization indexes, $\bar{N}=64 N$. Symbols denote FTS results, solid curves are ROL predictions, and the dashed line is the RPA prediction. The inset compares the nonlinear dependence of $\chi$ on $\chi_{b}$, eq 5 , with the linear approximation, $\chi \approx z_{\infty} \chi_{b}$.

\section{Order-Disorder Transition}

We now reexamine the ODT estimated previously by MC-FTS for $N=16,28$ and $64,{ }^{19}$ but this time using L-FTS with much larger simulation boxes. As before, the equilibrium lamellar period is estimated by $D \approx 2 \pi / k^{*}$, where $k^{*}$ is the peak of the disordered-state structure function, $S(k)$, extrapolated to the expected ODT in eq 4. Using the revised peaks, this gives $D / a \approx 6.28,8.11$, and 11.91 for $N=16,28$ and 64 , respectively.

The previous estimates of the ODT used parallel tempering, ${ }^{35}$ where $M$ simulations are performed in parallel over a sequence of interaction parameters, $\chi_{b}^{(i)}$, spanning the expected position of the transition. At intervals of $10^{3} \delta \tau$, the configurations $W_{-}^{(i)}(\mathbf{r})$ and $W_{-}^{(i+1)}(\mathbf{r})$ of 
the replicas at $\chi_{b}^{(i)}$ and $\chi_{b}^{(i+1)}$, respectively, are swapped with a probability of

$$
\begin{aligned}
p_{\text {swap }}= & \min \left\{1, \exp \left[\left(\frac{\rho_{0}}{\chi_{b}^{(i)}}-\frac{\rho_{0}}{\chi_{b}^{(i+1)}}\right)\right.\right. \\
& \left.\left.\times \int\left(\left[W_{-}^{(i)}\right]^{2}-\left[W_{-}^{(i+1)}\right]^{2}\right) d \mathbf{r}\right]\right\}
\end{aligned}
$$

To take full advantage of the parallelization, swaps are attempted between all pairs of neighboring replicas simultaneously, alternating between odd and even values of $i$. The phase at each $\chi_{b}^{(i)}$ value is monitored by evaluating the order parameter $\langle\Psi\rangle$, where

$$
\Psi=\left(\frac{N}{V}\right)^{2} \max _{\mathbf{k}}\left|W_{-}(\mathbf{k})\right|^{2}
$$

is averaged over the last $10^{4} \delta \tau$. One parallel-tempering run is performed starting from disordered configurations, and then a second run is performed starting from ordered lamellar configurations generated during the first run. Comparing the two runs generally reveals a metastability interval, where the disordered and ordered phases both survive for the full duration of the simulation. The position of the interval brackets the true equilibrium ODT.

Figure 4a shows our new parallel tempering results for $N=16$. The previous MCFTS results ${ }^{19}$ from disorder and order produced statistically equivalent curves in a small simulation box, $m=12$, corresponding to two periods, which are consistent with our LFTS results (only the run from disorder is shown). Given the increased speed of L-FTS, we can now apply parallel tempering to larger boxes of $m=18$. The resulting run from disorder produces the lamellar configurations shown in Figure 5; the label $(h k l)$ denotes a lamellar phase with principle wavevector $\mathbf{k}^{*}=2 \pi(h, k, l) / m \Delta$. The (220) configuration has a period $1.3 \%$ above the expected value, while the (300) and (221) configurations have a period $4.5 \%$ below. Therefore, the run from order is seeded with equal numbers of (220) and (300) configurations. For this larger system, the runs from disorder and order produce a narrow but distinct metastability interval centered around $\chi_{b} N \approx 21$. L-FTS allow us to 

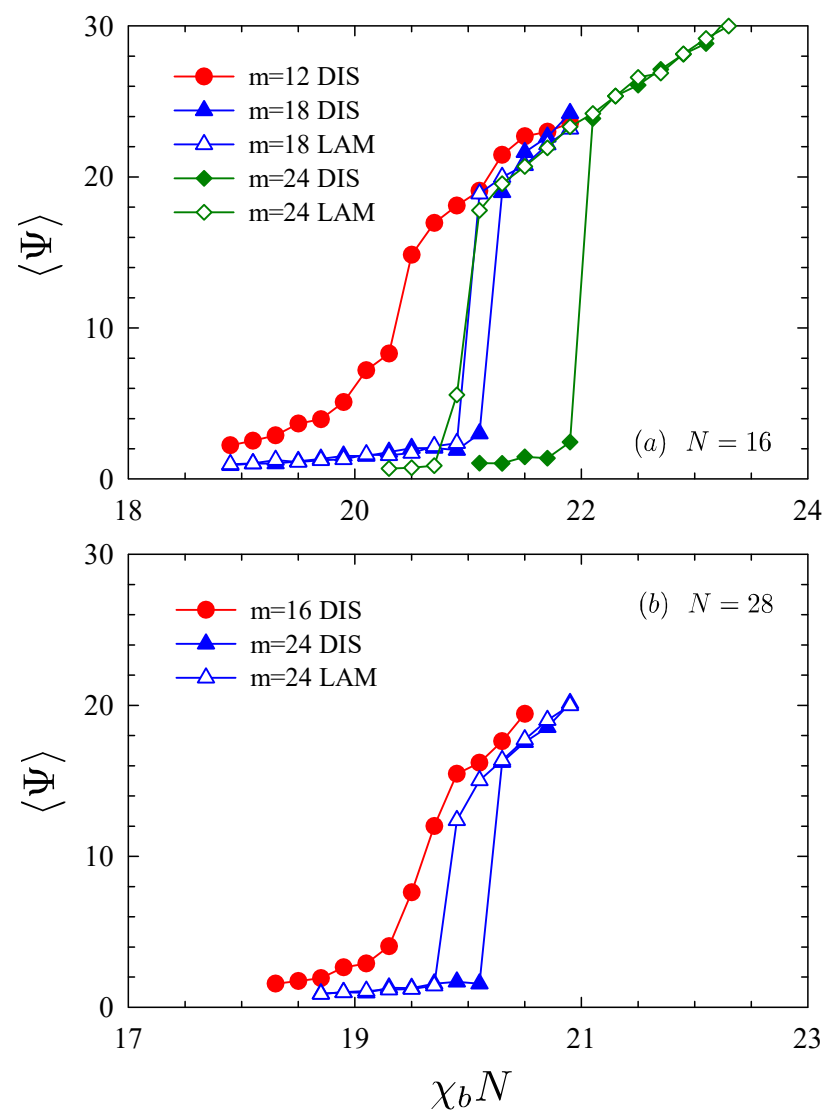

Figure 4: Order parameter, $\langle\Psi\rangle$, from parallel-tempering runs for molecules of polymerization (a) $N=16$ and (b) $N=28$ in simulation boxes of different sizes, $m$. Solid and open symbols denote runs initialized with disordered and ordered configurations, respectively.

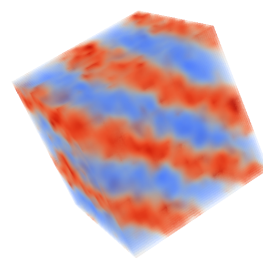

(220)

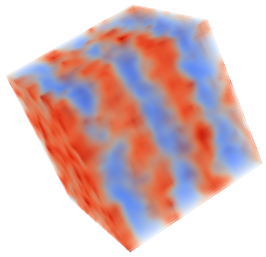

(300)

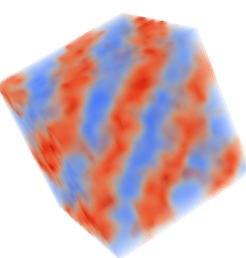

(221)

Figure 5: Lamellar configurations that spontaneously formed during the parallel-tempering run for $N=16$ in the $m=18$ simulation box. The lamellar period of a $(h k l)$ configuration is given by $D=m \Delta / \sqrt{h^{2}+k^{2}+l^{2}}$. 
consider even larger boxes of $m=24$. In this case, the run from order is seeded with (321) lamellae, the only configuration produced during the run from disorder and also the one that best matches the expected period. Not surprisingly, the metastability interval for $m=24$ is much wider and brackets the one for $m=18$.

Analogous results for the longer chains of $N=28$ are shown in Figure 4b. As before, ${ }^{19}$ the two-period box, $m=16$, fails to produce a discernible metastability interval. Therefore, we increase the box size to $m=24$. The disorder run mainly produces (300) and (221) configurations with a period $1.3 \%$ smaller than the expected value, but there is one (220) configuration with a period $4.6 \%$ larger than the expected value. Given the clear preference for the smaller period, the run from order is just seeded with (300) configurations. Again, the larger box size results in a well-defined metastability interval, locating the transition at $\left(\chi_{b} N\right)_{\mathrm{ODT}} \approx 20$

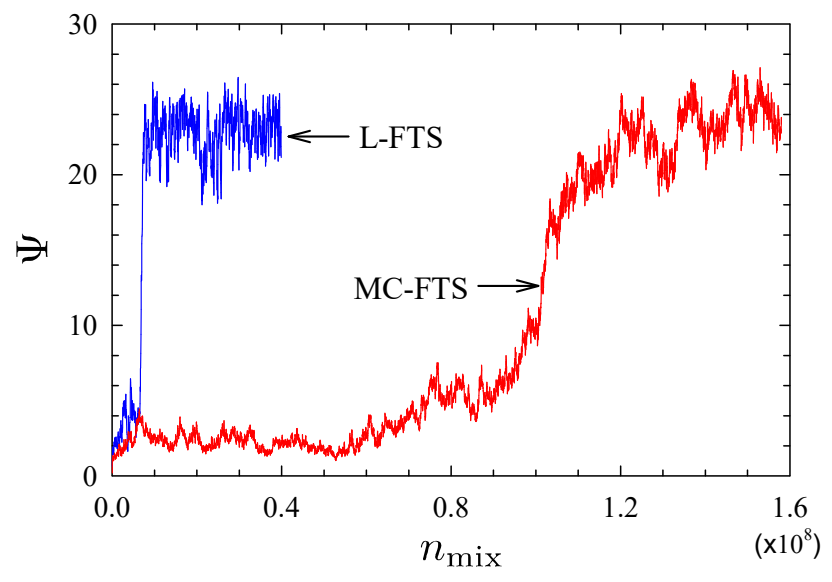

Figure 6: Speed comparison of L-FTS and MC-FTS showing the number of Anderson mixing iterations, $n_{\text {mix }}$, required for an initial field of $W_{-}(\mathbf{r})=0$ to spontaneously order into a lamellar phase for chains of $N=16$ at $\chi_{b} N=21.9$ in a simulation box of $m=18$.

To understand why the previous MC-FTS ${ }^{19}$ were unsuccessful for the larger boxes, Figure 6 compares the computational time for an initial field of $W_{-}(\mathbf{r})=0$ to spontaneously order into a stable lamellar phase at $\chi_{b} N=21.9$. Even in a relatively small box of $m=18$, the L-FTS produce the ordered phase approximately 20 times faster than the MC-FTS; the difference becomes even more pronounced for larger simulation boxes. Nevertheless, even 
with the much improved speed, the finite widths of the metastability intervals in Figure 4 seriously limit the resolution of our ODT measurements.

We address this problem by employing thermodynamic integration. The first step is to determine the free energy of the polymeric system relative to a reference system of known free energy. For this, we use an Einstein crystal consisting of independent harmonic oscillators, ${ }^{13}$ with the Hamiltonian

$$
\frac{H_{\mathrm{ec}}\left[W_{-}\right]}{k_{B} T}=\frac{\rho_{0}}{\chi_{b}^{*}} \int\left[W_{-}(\mathbf{r})-W_{0}(\mathbf{r})\right]^{2} d \mathbf{r}
$$

where $\chi_{b}^{*}$ is our estimate of the ODT and $W_{0}(\mathbf{r})$ is a reference field used to select the desired phase. We set $W_{0}(\mathbf{r})=0$ to obtain the disordered phase and $W_{0}(\mathbf{r}) \propto \sin (\mathbf{k} \cdot \mathbf{r})$ to obtain a lamellar phase of wavevector, k. (Note that the free energy of the Einstein crystal is independent of $W_{0}(\mathbf{r})$.) The composite Hamiltonian

$$
H\left[W_{-}\right]=\lambda H_{\mathrm{p}}\left[W_{-}, w_{+}\right]+(1-\lambda) H_{\mathrm{ec}}\left[W_{-}\right]
$$

is then simulated by changing the forcing term to

$$
g_{-}\left[W_{-}, w_{+}\right]=\lambda \phi_{-}\left[W_{-}, w_{+}\right]+\frac{2 \lambda}{\chi_{b}} W_{-}+\frac{2(1-\lambda)}{\chi_{b}^{*}}\left(W_{-}-W_{0}\right)
$$

To obtain the free energy of the composite system relative to the Einstein crystal, $F(\lambda)$, we simply integrate the derivative

$$
\frac{d F}{d \lambda}=\left\langle H_{\mathrm{p}}\left[W_{-}, w_{+}\right]\right\rangle-\left\langle H_{\mathrm{ec}}\left[W_{-}\right]\right\rangle
$$

This is done using continuous thermodynamic integration, ${ }^{36}$ where the integration and ensemble averaging is performed simultaneously by incrementing $\lambda$ between successive Langevin steps. Figure 7a shows $F(\lambda)$ obtained from MC-FTS and L-FTS (solid curves), using the same relatively large step size of $d \lambda=10^{-3}$. Naturally, $F(\lambda)$ approaches an asymptotic limit (dashed curve) as $d \lambda \rightarrow 0$. Noting that the error accumulates faster at large $\lambda$, our 
accurate estimates of the relative free energy of the polymeric system, $F(1)$, are evaluated using $d \lambda=10^{-6}$ for $\lambda<0.9$ and switching to $d \lambda=10^{-7}$ for $\lambda>0.9$. Figure $7 \mathrm{~b}$ compares the error, $\Delta F$, at the end of the thermodynamic integration for L-FTS (solid symbols) and MC-FTS (open symbols) as a function of $d \lambda$. To achieve an equivalent level of accuracy, the MC-FTS requires $d \lambda$ to be about 30 times smaller, which slows down the simulations by a similar factor. This explains why previous efforts to use thermodynamic integration with MC-FTS were unsuccessful. ${ }^{19}$ With L-FTS, we can now readily obtain accuracies of $\Delta F / n k_{B} T \lesssim 10^{-4}$.
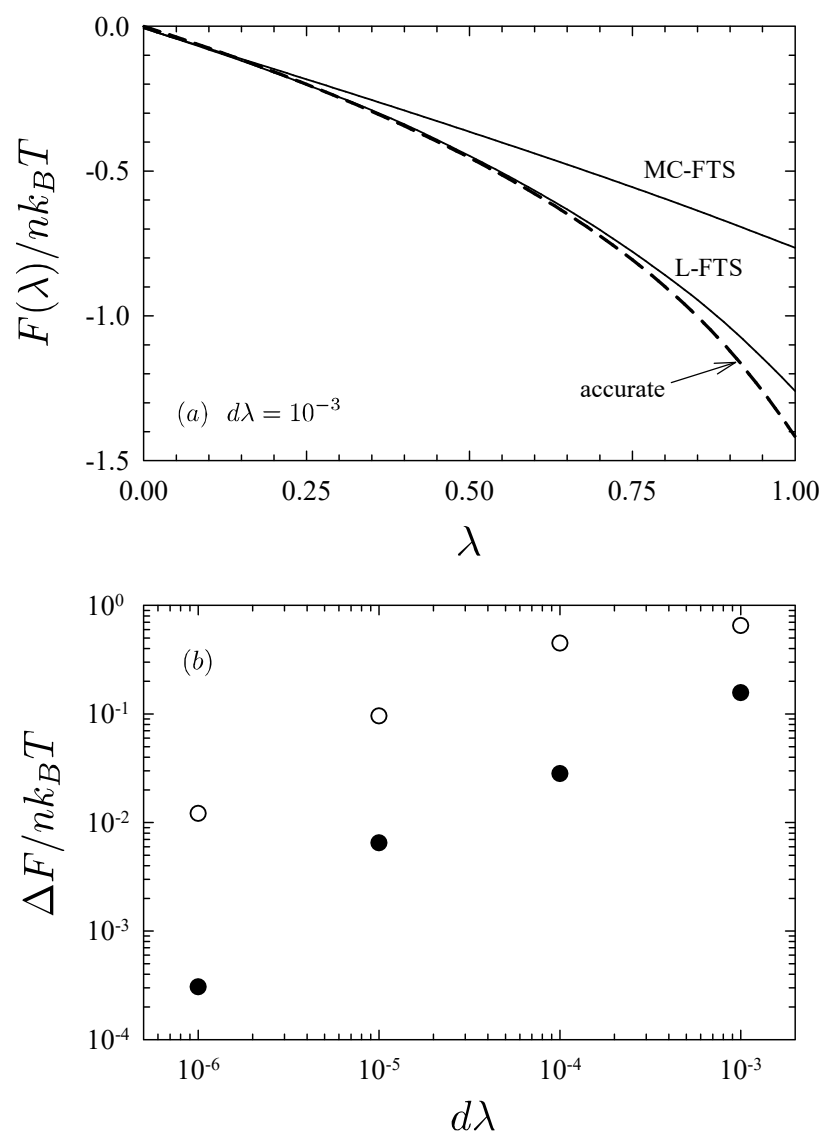

Figure 7: (a) Free energy, $F(\lambda)$, of the composite $H\left[W_{-}\right]$in eq 21 calculated by L-FTS and MC-FTS with an integration step of $d \lambda=10^{-3}$ compared to a highly accurate result for $N=16, \chi_{b} N=20$ and $m=18$. (b) Absolute error, $\Delta F$, at $\lambda=1$ as a function of step size, $d \lambda$, obtained with L-FTS (closed symbols) and MC-FTS (open symbols).

Once we have the relative free energy of the disordered phase, $F_{\mathrm{DIS}}$, at a $\chi_{b}$ below the 
ODT, it is integrated in the positive $\chi_{b}$ direction using

$$
\frac{d F}{d \chi_{b}}=-\frac{k_{B} T \rho_{0}}{\chi_{b}^{2}} \int\left\langle W_{-}^{2}(\mathbf{r})\right\rangle d \mathbf{r}
$$

with a small step size of $d \chi_{b}=10^{-6} / N$, which provides a similar accuracy to that of the $\lambda$ integrations. Likewise, the relative free energy of the lamellar phase, $F_{\mathrm{LAM}}$, is integrated in the negative $\chi_{b}$ direction, starting from a point above the ODT. Figure 8 plots the difference in the two free energies as a function of $\chi_{b} N$. The kinks at small $\chi_{b} N$ result when the backward run for the lamellar phase disorders, and the kinks at large $\chi_{b} N$ result when the forward run for the disordered phase spontaneously orders. Thus, it is only in the intervening interval that actually corresponds to the free energy difference between the disordered and lamellar phases. The flat regions to either side occur because both phases are the same, and the free energy is displaced from zero because the thermodynamic integration is unable to follow the change in free energy through a discontinuous phase transition. The fact that the slopes, obtained from continuous thermodynamic integrations of the same phase in opposite directions, are so close to zero testifies to the high accuracy of the $\chi_{b}$ integrations. ${ }^{36}$ There are, nevertheless, a couple of instances where the slope for the two lamellar phases is slightly positive or negative, but this is only because they happen to have different periods.

Our results for the short $N=16$ polymers in Figure 8a show that the (300) lamellar configuration is slightly favored over the (220) one in the $m=18$ simulation box, which is indeed consistent with the expected period, $D \approx 2 \pi / k^{*}$. Furthermore, the ODT (i.e., $\left.F_{\mathrm{LAM}}=F_{\mathrm{DIS}}\right)$ nicely matches the metastability interval in Figure 4a from parallel tempering. With thermodynamic integration, however, we can now obtain an accurate estimate of the ODT for the larger system of $m=24$. As it turns out, this only results in a slight shift to smaller $\left(\chi_{b} N\right)_{\mathrm{ODT}}$, implying that boxes of $L \gtrsim 3 D$ are adequate. Similar results for $N=28$ are presented in Figure 8b. This time, the (300) lamellar configuration is significantly preferred over the (220) one in the $m=24$ box, which is again consistent with the parallel 

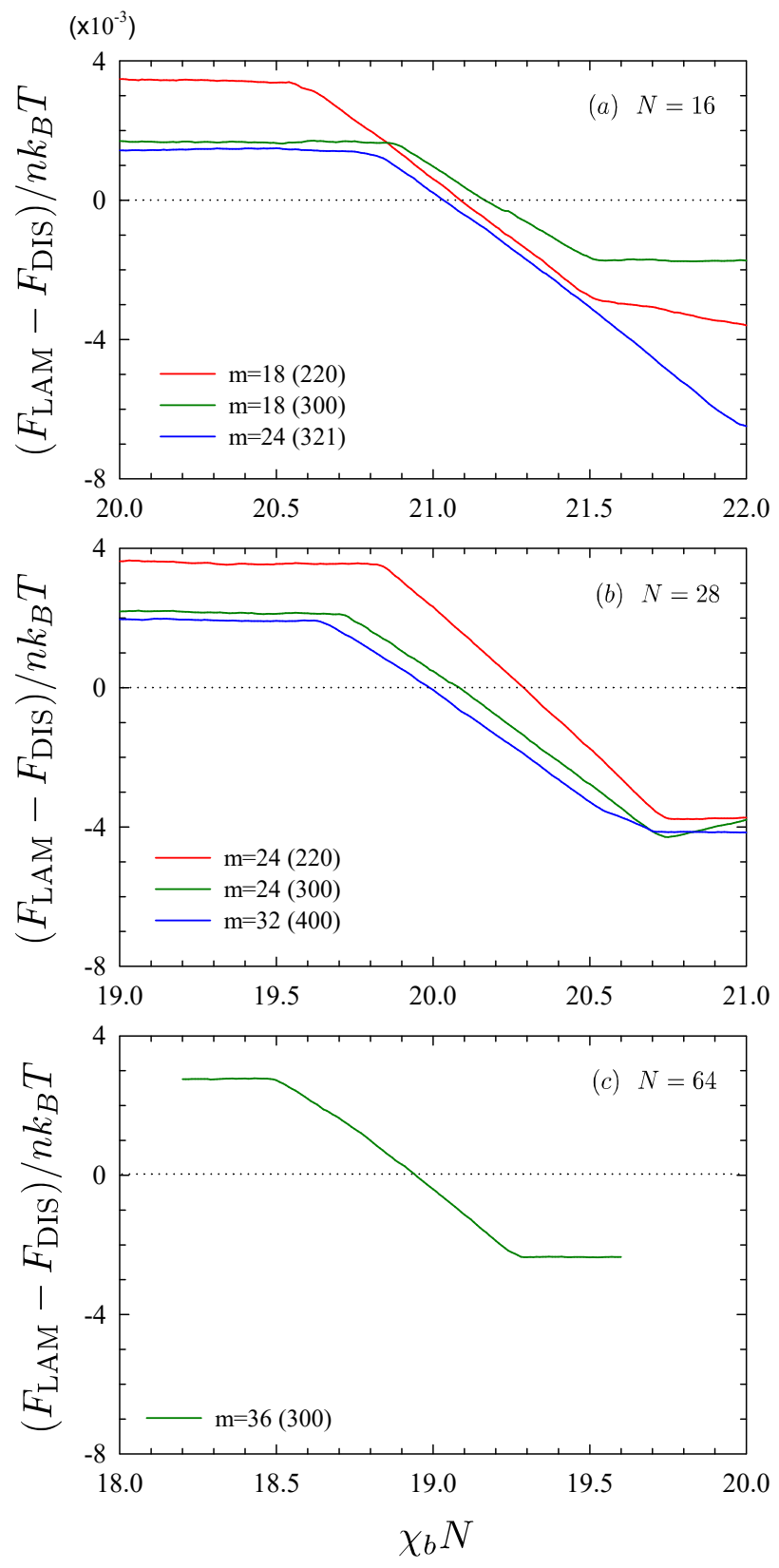

Figure 8: Free energy difference between lamellar and disordered phases, $F_{\mathrm{LAM}}-F_{\mathrm{DIS}}$, from thermodynamic integration for molecules of polymerization (a) $N=16$, (b) $N=28$, and (c) $N=64$ for different lamellar orientations, $(h k l)$, in simulation boxes of different sizes, $m$. The kinks at small and large $\chi_{b} N$ result when the metastable phase switches to the stable phase. 
tempering. As before, the ODT shifts to slightly smaller $\left(\chi_{b} N\right)_{\mathrm{ODT}}$, when the system size is increased. Now that we have a good gauge of the finite-size effects, we only perform one set of thermodynamic integrations for $N=64$ in Figure 8c, using a $m=36$ box and a (300) lamellar configuration that is within $1 \%$ of the expected period.

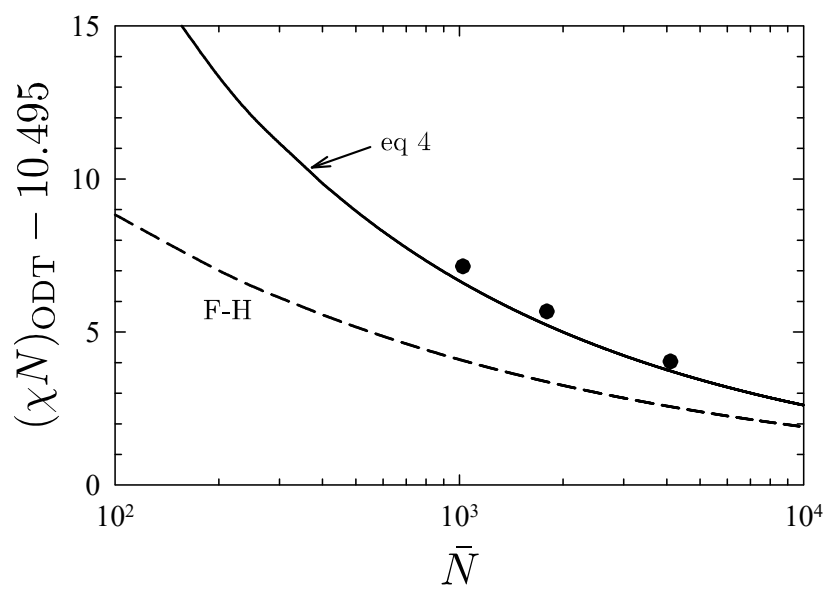

Figure 9: Fluctuation correction to the ODT of symmetric diblock copolymer melts plotted in terms of the effective $\chi$ and the invariant polymerization index, $\bar{N}$. The solid curve denotes the universal result from particle-based simulations, eq $4,{ }^{10}$ and the dashed curve is the Fredrickson-Helfand prediction. ${ }^{9}$

Based on the thermodynamic integrations for our largest system sizes, we estimate the ODT for $N=16,28$, and 64 to be $\left(\chi_{b} N\right)_{\mathrm{ODT}}=21.0,20.0$, and 18.9, respectively. These estimates are plotted in Figure 9 in terms of the effective $\chi$ in eq 5 and the invariant polymerization index $\bar{N}=64 N$. The L-FTS agree well with the universal results from particle-based simulations (solid curve), much better so than the Fredrickson-Helfand prediction (dashed curve).

\section{Discussion}

We have conducted the first head-to-head comparison of MC-FTS and L-FTS, illustrating that simulations that would have taken months by MC-FTS can now be completed in a few days by L-FTS. This efficiency was not recognized previously, because it also relies on the algorithms for the diffusion equation and the partial saddle-point approximation. The origi- 
nal L-FTS of Reister et al. ${ }^{20}$ only managed $7^{3}$ wavevectors, because they solved the diffusion equation with a spectral method and the saddle point with a Jacobian-based algorithm. Alexander-Katz and Fredrickson ${ }^{21}$ performed L-FTS in simulation boxes with $48 \times 48 \times 16$ grid points, but only for relatively weak fluctuations corresponding to $\bar{N}=5.4 \times 10^{5}$ and a far lower error tolerance for $w_{+}(\mathbf{r})$. They implemented the pseudo-spectral method without Richardson extrapolation and located the saddle point with a simple mixing iteration. Key to the current performance is our use of Richardson extrapolation and Anderson mixing. ${ }^{31}$ Naturally, we can expect further improvements in the future, as L-FTS gains more attention.

The universality hypothesis for block copolymer phase behavior ${ }^{5,6}$ implies that the $(\chi N)$ ODT values from our L-FTS should match the universal curve in eq 4 obtained from particle-based simulations. ${ }^{10}$ Indeed, Figure 9 shows that it does capture the vast majority of the fluctuation correction to the SCFT prediction, $(\chi N)_{\text {ODT }}=10.495$. Nevertheless, there is a noticeable discrepancy of up to 0.5 for our smallest $\bar{N} \approx 10^{3}$. Given the slopes of the free energy differences in Figure 8, the contribution due to inaccuracies in the thermodynamic integration should be less than 0.02. The contribution due to finite-size effects is larger but still presumably below 0.1 , based on, for example, the spread among the ODTs for the $N=16$ polymers in Figure 8a. Thus, the discrepancy undoubtedly results from something else.

One potential explanation for the discrepancy is the finite grid spacing relative to the width of the internal $\mathrm{A} / \mathrm{B}$ interfaces, which is estimated by $w_{I} \approx 2 a / \sqrt{6 \chi} \cdot{ }^{37}$ For our shortest $N=16$ chains, $w_{I} \approx 0.8 a$ is comparable to $\Delta=a$. As $N$ increases, the value of $\chi$ at the ODT decreases resulting in wider interfaces, and thus the discrepancy should diminish with increasing $\bar{N}$, as it appears to do so in Figure 9. Another source of discrepancy is the partial saddle-point approximation for $W_{+}(\mathbf{r})$, which becomes increasingly inaccurate as $\bar{N}$ deviates from infinity. Although past comparisons with CL-FTS have shown it to be accurate, ${ }^{15,21}$ they have not considered the level of fluctuations in the present study. The clearest evidence of inaccuracy in our study is the inability of L-FTS to capture the departure of $S\left(k^{*}\right)$ from the RPA prediction at small $\chi N$ in Figure 3. Even still, this inaccuracy remains relatively 
small for $\bar{N} \gtrsim 10^{3}$.

Although partial saddle-point approximation can, in principle, be avoided with CL-FTS, this approach has its own challenges. In addition to the UV divergence, CL-FTS for the standard Gaussian chain model (GCM) experience an instability that prevents their application to realistic values of $\bar{N}{ }^{38}$ Delaney and Fredrickson ${ }^{14}$ remove the UV divergence and the instability by introducing compressibility and a finite-range to the interactions. This, however, requires a calibration in order to map the modified model back onto the standard GCM. They mention the possibility of performing a Morse calibration, but leave it for a future calculation. However, to obtain universal behavior, the range of the interactions cannot be too large relative to the width of the $\mathrm{A} / \mathrm{B}$ interfaces, $w_{I}$. It remains to be seen if CL-FTS can be performed at realistic values of $\bar{N}$, while keeping the interactions sufficiently short range. In any case, even with the partial saddle-point approximation, our estimate of the fluctuation correction in Figure 9 is accurate to well within 10\%, which is already a considerable improvement over the Fredrickson-Helfand prediction. ${ }^{9}$

A convenient feature of L-FTS are their similarity to SCFT calculations, on account of the fact that they rely on the same statistical mechanics of non-interacting polymers. Consequently, the conversion of SCFT code to L-FTS code is trivial. Instead of solving $2 \mathrm{~m}^{3}$ equations (i.e., $\left.g_{-}\left[w_{-}, w_{+}\right]=g_{+}\left[w_{-}, w_{+}\right]=0\right)$ for the full saddle point, $w_{-}(\mathbf{r})$ and $w_{+}(\mathbf{r})$, one just solves $m^{3}$ equations (i.e., $\left.g_{+}\left[W_{-}, w_{+}\right]=0\right)$ for the partial saddle point, $w_{+}(\mathbf{r})$, and evolves $W_{-}(\mathbf{r})$ using Langevin dynamics with $g_{-}\left[W_{-}, w_{+}\right]$as the forcing term.

Although conventional particle-based simulations can already handle diblock copolymer melts over the full experimental range of $\bar{N}, 7,10,39$ this is not generally the case for more complicated systems. On the other hand, as explicitly demonstrated in previous studies, field-theoretic simulations can be readily extended to elaborate block copolymers architectures $^{40,41}$ and innovative ensembles for dealing with blends. ${ }^{42-44}$ For AB-type systems, this just involves changing the $n \ln Q$ term in eq 1 for the Hamiltonian from that of non-interacting diblock copolymers in a canonical ensemble to the appropriate expression for the system of 
interest in the ensemble of interest. Of course, this also involves modifications to eqs 9 and 10 for the composition, $\phi_{-}(\mathbf{r})$, and total concentration, $\phi_{+}(\mathbf{r})$, respectively, but that is also relatively trivial. The extension to three or more chemically-distinct components (e.g., ABC-type melts) is more involved, ${ }^{33}$ but certainly not overly onerous.

\section{Summary}

This study has presented an algorithm for field-theoretic simulations (FTS), capable of handling the level of fluctuations typical of experiment. ${ }^{26}$ It evolves the composition field, $W_{-}(\mathbf{r})$, using conventional Langevin dynamics while approximating the pressure field with its saddle point, $w_{+}(\mathbf{r}) .{ }^{20}$ The saddle point is located using Anderson mixing and the single-chain statistical mechanics is solved using a pseudo-spectral method with Richardson extrapolation. ${ }^{31}$ This Langevin approach (L-FTS) proves to be much faster than the previous Monte Carlo approach (MC-FTS) used in ref 19, which allows for far superior statistics and considerably larger simulation boxes.

The algorithm was demonstrated on symmetric diblock copolymer melts with invariant polymerization indexes of $\bar{N} \sim 10^{3}$. We first improved the Morse calibration ${ }^{5,23}$ of the effective $\chi$ parameter, shown in the inset of Figure 3, and then evaluated the ODT for $N=16,28$, and 64 using parallel tempering and thermodynamic integration. Both methods gave consistent predictions for the ODT, but the latter provided the more accurate results plotted in Figure 9. Based on our assessment of statistical inaccuracies and finite-size effects, our estimates of the ODT should be accurate to within the symbol size used in the plot. This leaves a small but significant deviation from the universal curve, eq 4, obtained from particle-based simulations. ${ }^{10}$ One potential source of the deviation is the finite size of our grid, $\Delta$, relative to the estimated width of the internal $\mathrm{A} / \mathrm{B}$ interfaces, $w_{I}$. Another is the partial saddle-point approximation for the pressure field.

Despite this, the L-FTS predicts the fluctuation corrections in Figure 9 to an accuracy 
of better than 10\%. The attractiveness of this approach is the ease with which SCFT calculations can be switched to L-FTS. However, the most significant merit of L-FTS is the advantage they have over particle-based simulations to consider blends and complicated block copolymer architectures. Given this, coupled with their remarkable efficiency, we anticipate that L-FTS will become the method of choice for evaluating fluctuation corrections to SCFT.

\section{Acknowledgments}

This work was supported by NSERC of Canada and computer resources were provided by Compute Canada.

\section{References}

(1) Helfand, E. Theory of Inhomogeneous Polymers - Fundamentals of Gaussian RandomWalk Model. J. Chem. Phys 1975, 63, 999-1005.

(2) Fredrickson, G. H. The Equilibrium Theory of Inhomogeneous Polymers; Oxford University Press: New York, 2006.

(3) Matsen, M. W. In Soft Matter: Polymer Melts and Mixtures; Gompper, G., Schick, M., Eds.; Wiley-VCH: Weinheim, Germany, 2006.

(4) Matsen, M. W. The Standard Gaussian Model for Block Copolymer Melts. J. Phys.: Condens. Matter 2001, 14, R21-R47.

(5) Glaser, J.; Qin, J.; Medapuram, P.; Müller, M.; Morse, D. C. Test of a Scaling Hypothesis for the Structure Factor of Disordered Diblock Copolymer Melts. Soft Matter 2012, 8, 11310-11317.

(6) Glaser, J.; Medapuram, P.; Morse, D. C. Collective and Single-Chain Correlations 
in Disordered Melts of Symmetric Diblock Copolymers: Quantitative Comparison of Simulations and Theory. Macromolecules 2014, 47, 851-869.

(7) Medapuram, P.; Glaser, J.; Morse, D. C. Universal Phenomenology of Symmetric Diblock Copolymers near the Order-Disorder Transition. Macromolecules 2015, 48, 819839.

(8) Yadav, M.; Bates, F. S.; Morse, D. C. Network Model of the Disordered Phase in Symmetric Diblock Copolymer Melts. Phys. Rev. Lett. 2019, 121, 127802.

(9) Fredrickson, G. H.; Helfand, E. Fluctuation Effects in the Theory of Microphase Separation in Block Copolymers. J. Chem. Phys. 1987, 87, 697-705.

(10) Glaser, J.; Medapuram, P.; Beardsley, T. M.; Matsen, M. W.; Morse, D. C. Universality of Block Copolymer Melts. Phys. Rev. Lett. 2014, 113, 068302.

(11) Ganesan V.; Fredrickson, G. H. Field-Theoretic Polymer Simulations. Europhys. Lett. 2001, 55, 814-820.

(12) Fredrickson, G. H.; Ganesan, V.; Drolet, F. Field-Theoretic Computer Simulation Methods for Polymers and Complex Fluids. Macromolecules 2002, 35, 16-39.

(13) Lennon, E. M.; Katsov, K.; Fredrickson, G. H. Free Energy Evaluation in FieldTheoretic Polymer Simulations. Phys. Rev. Lett. 2008, 101, 138302.

(14) Delaney, K. T.; Fredrickson, G. H. Recent Developments in Fully Fluctuating FieldTheoretic Simulations of Polymer Melts and Solutions. J. Phys. Chem. B 2016, 120, $7615-7634$.

(15) Düchs, D.; Ganesan, V.; Fredrickson, G. H.; Schmid, F. Fluctuation Effects in Ternary AB+A+B Polymeric Emulsions. Macromolecules 2003, 36, 9237-9248. 
(16) Düchs, D.; Schmid, F. Formation and Structure of the Microemulsion Phase in TwoDimensional Ternary AB+A+B Polymeric Emulsions. J. Chem. Phys. 2004, 121, 27982805.

(17) Stasiak P.; Matsen, M. W. Monte Carlo Field-Theoretic Simulations for Melts of Symmetric Diblock Copolymer. Macromolecules 2013, 46, 8037-8045.

(18) Vorselaars, B.; Stasiak, P.; Matsen, M. W. Field-Theoretic Simulation of Block Copolymers at Experimentally Relevant Molecular Weights. Macromolecules 2015, 46, 90719080.

(19) Beardsley, T. M.; Matsen, M. W. Calibration of the Flory-Huggins Interaction Parameter in Field-Theoretic Simulations. J. Chem. Phys. 2019, 150, 174902.

(20) Reister, E.; Müller, M.; Binder, K. Spinodal Decomposition in a Binary Polymer Mixture: Dynamic Self-Consistent-Field Theory and Monte Carlo Simulations. Phys. Rev. E 2001, 64, 041804 .

(21) Alexander-Katz, A.; Fredrickson, G. H. Diblock Copolymer Thin Films: A FieldTheoretic Simulation Study. Macromolecules 2007, 40, 4075-4087.

(22) Olvera de la Cruz, M.; Edwards, S. F.; Sanchez, I. C. Concentration Fluctuations in Polymer Blend Thermodynamics. J. Chem. Phys. 1988, 89, 1704-1708.

(23) Qin, J.; Morse, D. C. Renormalized One-Loop Theory of Correlations in Polymer Blends. J. Chem. Phys. 2009, 130, 224902.

(24) Grzywacz, P.; Qin, J.; Morse, D. C. Renormalization of the One-Loop Theory of Fluctuations in Polymer Blends and Diblock Copolymer Melts. Phys. Rev E 2007, 76, 061802.

(25) Qin, J.; Grzywacs, P.; Morse, D. C. Renormalized One-Loop Theory of Correlations in Disordered Diblock Copolymers. J. Chem. Phys. 2011, 135, 084902. 
(26) Bates, F. S.; Schulz, M. F.; Khandpur, A. K.; Förster, S.; Rosedale, J. H. Fluctuations, Conformational Asymmetry and Block Copolymer Phase Behaviour. Faraday Discuss. 1994, 98, 7-18.

(27) Rasmussen, K. O Kalosakas, G. Improved Numerical Algorithm for Exploring Block Copolymer Mesophases. J. Polym. Sci., Part B 2002, 40, 1777-1783.

(28) Ranjan, A.; Qin, J.; Morse, D. C. Linear Response and Stability of Ordered Phases of Block Copolymer Melts. Macromolecules 2008, 41, 942-954.

(29) Anderson, D. G. Iterative Procedures for Nonlinear Integral Equations. J. Assoc. Comput. Mach. 1965, 12, 547-560.

(30) Thompson, R. B.; Rasmussen, R. O.; Lookman, L. Improved Convergence in Block Copolymer Self-Consistent Field Theory by Anderson Mixing. J. Chem. Phys. 2004, 120, 31-34.

(31) Stasiak, P.; Matsen, M. W. Efficiency of Pseudo-Spectral Algorithms with Anderson Mixing for the SCFT of Periodic Block-Copolymer Phases. Eur. Phys. J. E 2011, 34, 110.

(32) For our particular case, we set the $d_{j}^{(k)}$ in eq 12 of ref 31 to the value of $g_{+}\left[W_{-}, w_{+}\right]$ evaluated at the $j$ 'th mesh point.

(33) Düchs, D.; Delaney, K. T.; Fredrickson, G. H. A Multi-Species Exchange Model for Fully Fluctuating Polymer Field Theory Simulations. J. Chem. Phys., 2014, 36, 174103.

(34) Leibler, L. Theory of Microphase Separation in Block Copolymers. Macromolecules 1980, 13, 1602-1617.

(35) Earl, D. J.; Deem, M. W. Parallel Tempering: Theory, Applications, and New Perspectives. Phys. Chem. Chem. Phys. 2005, 7, 3910-3916. 
(36) Spencer, R. K. W.; Vorselaars, B.; Matsen, M. W. Continuous Thermodynamic Integration in Field-Theoretic Simulations of Structured Polymers. Macromol. Theory Simul. 2017, 26, 1700036 .

(37) Semenov, A. N. Contribution to the Theory of Microphase Layering in Block-Copolymer Melts. Sov. Phys. JETP 1985, 61, 733-742.

(38) Koski, J.; Chao, H.; Riggleman, R. A. J. Field Theoretic Simulations of Polymer Nanocomposites. J. Chem. Phys. 2013, 139, 244911.

(39) Willis, J. D.; Beardsley, T. M.; Matsen, M. W. Calibration of a Lattice Model for High-Molecular-Weight Block Copolymer Melts. J. Chem. Phys. 2019, 150, 204906.

(40) Liu, Y.-X.; Delaney, K. T.; Fredrickson, G. H. Field-Theoretic Simulations of Fluctuation-Stabilized Aperiodic 'Bricks-and-Mortar' Mesophase in Miktoarm Star Block Copolymer/Homopolymer Blends. Macromolecules 2017, 50, 6263-6272.

(41) Spencer, R. K. W.; Matsen, M. W. Field-Theoretic Simulations of Bottlebrush Copolymers. J. Chem. Phys. 2018, 149, 184901.

(42) Riggleman, R. A.; Fredrickson, G. H. Field-Theoretic Simulations in the Gibbs Ensemble. J. Chem. Phys. 2010, 132, 024104.

(43) Koski, J. P.; Riggleman, R. A. Field-Theoretic Simulations of Block Copolymer Nanocomposites in a Constant Interfacial Tension Ensemble. J. Chem. Phys. 2017, $146,164903$.

(44) Spencer, R. K. W.; Matsen, M. W. Fluctuation Effects in Blends of A+B Homopolymers with AB Diblock Copolymer. J. Chem. Phys. 2018, 148, 204907. 


\section{for Table of Contents use only}

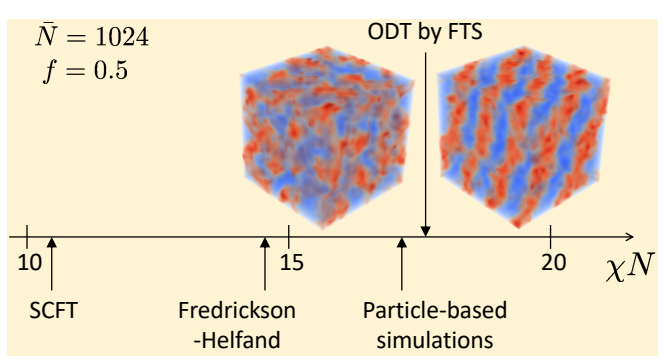

Title: Computationally Efficient Field-Theoretic Simulations for Block Copolymer Melts. Authors: T. M. Beardsley, R. K. W. Spencer, and M.W. Matsen 